\title{
Urothelial bladder carcinoma metastasizing to the eye: A systematic review and case report
}

\author{
GUIDO GIORDANO $^{1-3^{*}}$, NUNZIO OLIVIERI ${ }^{2 *}$, MARIO ROSARIO D'ANDREA $^{3}$, TANIA DI RAIMO $^{3}$, \\ ERMINIA MANFRIN ${ }^{4}$, ANDREA REMO $^{5}$, MASSIMO PANCIONE $^{6}$, MATTEO BRUNELLI $^{4}$, ELENA DE SANTIS $^{7}$, \\ GIULIA COPPOLA ${ }^{7}$, ALDO SCARPA $^{4-8}$, LUIGI COPPOLA $^{9}$ and PIETRO PARCESEPE ${ }^{4}$ \\ ${ }^{1}$ Oncology Division IRCCS Casa Sollievo della Sofferenza, San Giovanni Rotondo, I-71013 Foggia; \\ ${ }^{2}$ Biology Department, Federico II University of Naples, I-80134 Naples; ${ }^{3}$ Medical Oncology Unit, San Filippo Neri Hospital, \\ I-00135 Rome; ${ }^{4}$ Department of Diagnostics and Public Health-Section of Pathology, University and Hospital Trust of Verona, \\ I-37134 Verona; ${ }^{5}$ Pathology Unit, Mater Salutis Hospital, ULSS9, Legnago, I-37045 Verona; ${ }^{6}$ Department of
}

Sciences and Technologies, University of Sannio, I-82100 Benevento; ${ }^{7}$ Department of Human Anatomy, Sapienza University of Rome, I-00185 Rome; ${ }^{8}$ ARC-Net Centre for Applied Research on Cancer, University and Hospital Trust of Verona, I-37134 Verona; ${ }^{9}$ UOC of Pathologic Anatomy, San Filippo Neri Hospital, I-00135 Rome, Italy

Received March 5, 2018; Accepted August 23, 2018

DOI: $10.3892 / 01.2018 .9579$

\begin{abstract}
The eye is a rare site for disseminated malignancies; nevertheless, several tumors may metastasize to ocular structures. Few cases of urothelial and bladder cancer with eye involvement have been described in the literature thus far. The rarity of metastatic ocular localization implies an accurate differential diagnosis among the possible primary tumor sites. However, a specific diagnostic algorithm is not currently available, nor a defined therapeutic approach. Eye metastases are associated with advanced disease and poor prognosis. Physicians should be made aware of the possibility of eye involvement in patients with a past medical history of
\end{abstract}

Correspondence to: Dr Pietro Parcesepe, Department of Diagnostics and Public Health-Section of Pathology, University and Hospital Trust of Verona, L.A. Scuro Square 10, I-37134 Verona, Italy

E-mail: parcesepe.pietro@gmail.com

Dr Guido Giordano, Oncology Division IRCCS Casa Sollievo della Sofferenza, Viale Cappuccini 1, San Giovanni Rotondo, I-71013 Foggia, Italy

E-mail: giordano.guido81@gmail.com

*Contributed equally

Abbreviations: NA, not available; M, male; F, female; L, left; R, right; B, bilateral; BSC, best supportive care; ADRIA, adriamycin; 5FU, 5-fluoroiracil; CDDP, cisplatin; CTX, cyclophosphamide; MVAC, methotrexate + vinblastine + adriamycin + cisplatin; CBDCA, carboplatin; GEM, gemcitabine; RT, radiotherapy; OS, overall survival; LF, lost to follow up; CT, computed tomography

Key words: urothelial carcinoma, bladder, ocular metastases, eye, GATA3 urothelial bladder cancer associated with ocular symptoms. The present case reports discusses the first documented case, to the best of our knowledge, of an urothelial bladder cancer metastasizing to the retro bulbar region that infiltrates the lacrimal gland. Furthermore, the report provides a systematic qualitative review of the current literature on eye metastases from urothelial bladder cancer using the Preferred Reporting Items for Systematic Reviews and Meta-Analyses.

\section{Introduction}

Bladder cancer is the ninth cause of tumor in the world and the second most common genitourinary malignancy. Urothelial carcinoma represents $90 \%$ of all primary bladder tumors (1). Half of patients affected by these tumors, will develop local recurrence or distant metastases after radical surgery and treatment in this setting remains exclusively palliative. Lymph nodes, liver, lung and bones represent the metastatic sites with higher incidence (2). The eye is a rare site for disseminated malignancies because of the absence of a lymphatic system and metastases may occur by haematogenous spread (3). Therefore, eye structures with the highest vascular supply are more likely affected, with an incidence from 1 to $13 \%$ (2). Breast cancer is the most common primary tumor metastasizing to the eye, followed in order of frequency by: Lung cancer, gastrointestinal tumors, and less commonly, thyroid, prostate, kidney, testicles, pancreatic, ovarian and liver cancer (4). Eye metastases comprise both orbital (bone, muscle and fat) and ocular (mainly uveal) localizations $(5,6)$. Majority of eye metastases in adults are located in the uvea and mainly in the choroid and orbital metastases are less frequent than uveal metastases (5). Generally, they onset as synchronous or metachronous localizations in patients with multiple metastatic sites and life expectancy is very poor. Twenty-three cases of urothelial or bladder tumors with eye metastases have been described in literature so far $(2,4-23)$. Here we report the first documented 
case, to our knowledge, of an urothelial-bladder cancer metastasizing to the retro-bulbar region and infiltrating the lacrimal gland. Furthermore, we provide a systematic qualitative review of the current literature on eye metastases from urothelial bladder cancer using the Preferred Reporting Items for Systematic Reviews and Meta-Analyses (PRISMA) (24). Finally, we aim to clarify the features, medical interventions, outcomes and we try to describe the natural course of the disease in this uncommon group of patients.

\section{Case report}

A 70 years old man came to the hospital in March 2017 because of visual disorders in the right eye, diplopia and diffuse pain in retro-bulbar region. His past medical history was characterized by chronic obstructive pulmonary disease (COPD) on treatment with Broncho-dilatators and arterial hypertension on treatment with ACE-inhibitor. In June 2014, patient had received radical cystectomy with lymphadenectomy for grade 3 , urothelial bladder cancer, stage pT4N0M0. Despite preoperative staging detected a muscle invasive cancer, the patient strongly preferred a surgical approach instead of neoadjuvant chemotherapy. After radical surgery, adjuvant chemotherapy with cisplatin plus gemcitabine combination was administered for 4 cycles. At the time of hospitalization, the patient was undergoing to a follow up program that was negative for both local recurrence and distant metastases up to six months before. Eye clinical examination detected any cystic neo-formation but evidenced reduced motility. At the abdomen palpation liver was at $2.5 \mathrm{~cm}$ from the right costal margin with an irregular surface. Complete blood count was within normal limits and biochemical evaluation showed liver impairment: Aspartate aminotransferase $470 \mathrm{U} / 1$, alanine aminotransferase $527 \mathrm{U} / 1$, gamma-glutamyl transferase 435 U/1. Contrast-enhanced computed tomography (CT) of the orbit showed an involvement of the right periorbital fat, retro bulbar spaces and lacrimal gland. Excisional biopsy was performed and samples from retro-bulbar fibro-adipose tissue and lacrimal gland were collected. Histological examination showed neoplastic infiltration of fibro adipose tissue characterized by diffuse population of cellular elements with a high eosinophilic cytoplasm and eccentric nuclei. Diffuse angiolymphatic invasion was also present. Immunohistochemistry stains were positive for GATA3, CKAE1/AE3, CK5, CK7, CK20, CD138, DNP63 and negative for LCA and CD79 $\alpha$ (Fig. 2). Finally, the histological examination was diagnostic for retro bulbar metastases from urothelial carcinoma. Subsequently, full body CT scan showed multiple liver metastases. Due to the patient's clinical condition (Eastern Cooperative Oncology Group-ECOG performance status 2) and the liver impairment evidenced by the biochemistry, no chemotherapy was administered and best supportive care was started. Unfortunately, because of widespread metastatic disease the patient died three months after the diagnosis of the ocular involvement.

Patient's medical history including comorbidities, concomitant medications, bladder cancer diagnosis, previous treatments and ocular metastasis detection were taken from clinical records. Written informed consent for the case publication was obtained from the patient at the time of retro-bulbar metastasis histological confirmation. The Ethics Committee approved all procedures.

Review criteria. A systematic review of the literature was performed in compliance with the PRISMA guidelines. Screening was performed by reviewing article titles or full text up to February 2018 using electronic database: MEDLINE and WEB OF SCIENCE. The primary search terms included 'ocular metastasis', 'eye metastasis' and 'urothelial bladder cancer' in the article titles. The extracted citations were then screened for duplicates. Later operators 'and' and 'or' were applied on the extracted records by use of the terms 'urothelial,' 'bladder', 'cancer', 'ocular', 'eye', 'metastasis' to narrow the scope of the review (Fig. 1). Twenty-four articles met eligibility criteria for our qualitative systematic review and 23 previously reported cases were identified. This population was described for median age, gender, ocular site, both systemic and ocular specific treatment. We also evaluated the time to ocular metastases, defined as the time elapsed between the urothelial bladder cancer diagnosis and the ocular metastases occurrence. Overall survival from ocular metastases was defined as the time elapsed from the eye involvement to death. Kaplan Meier analysis for survival was performed using GraphPad Prism 5.04 for Windows (GraphPad Software, Inc., La Jolla, CA, USA). This evaluation included only patients whose survival data were available.

\section{Discussion}

Eye metastases are uncommon, accounting for between 2.5 and $8.1 \%$ of all orbital space-occupying lesions (25). The majorities are adenocarcinoma and the most common primary site is breast (26). Prostate, colon, and lung cancer can also metastasize to the eye. Bladder carcinoma is an extremely rare source of eye metastases. Since the first case reported in 1965, only 23 additional cases of eye metastases from bladder cancer have been reported in literature prior to the present case. This case is extremely rare since the patient developed retro bulbar metastasis with lacrimal gland infiltration and it is the first described in literature. Seventy per cent of bladder tumors do not extend beyond the lamina propria at the time of diagnoses. Among the patients treated with radical cystectomy $57 \%$ are characterized by muscular infiltration while the remaining $43 \%$ show non-infiltrative disease that progress to an infiltrative carcinoma. Around a quarter of patients receiving radical surgery has lymph-nodes involvement and one third of the cases with infiltrative carcinoma could have micro metastatic disease at the time of primary tumor treatment (25). In the present case patient obtained cystectomy and lymphadenectomy three years before the occurrence of retrobulbar and liver metastases. The current state of the art for muscle invasive bladder cancer treatment is neoadjuvant, platinum-based chemotherapy. Nevertheless, in this case the patient chose an immediate surgical approach. At the time of diagnosis, he showed pT4N0M0, G3 urothelial bladder carcinoma. Despite of infiltrative disease no lymph nodes were involved at the time of surgery. Notably, the patient received adjuvant chemotherapy but he developed multiple liver and ocular metastases. Therefore, the concept of an aggressive, 


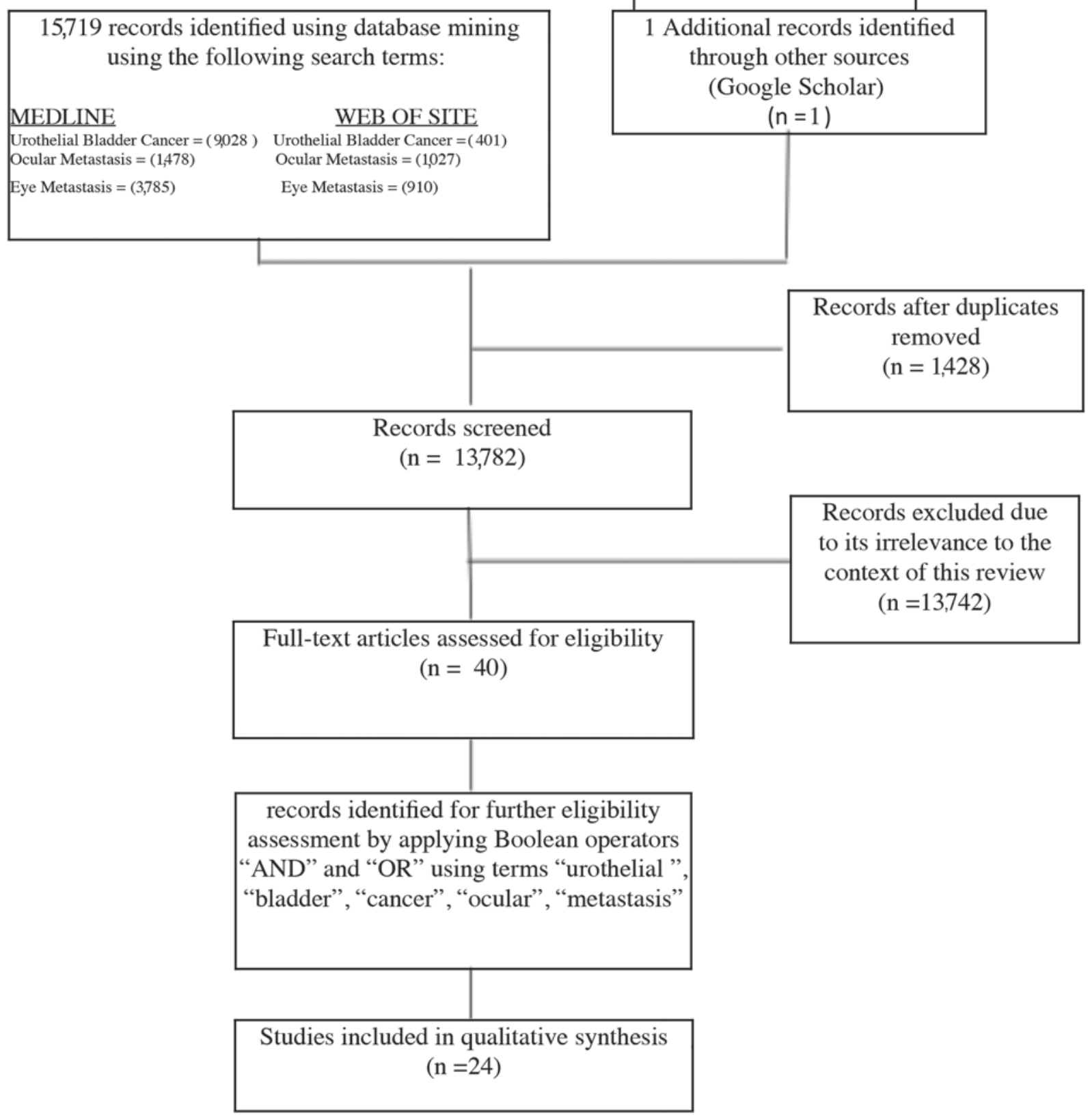

Figure 1. Preferred Reporting Items for Systematic Reviews and Meta-Analyses protocol used for the systematic review.

micro-metastatic disease from the beginning should be taken into account. Usually, bladder cancer metastasizes to lymph nodes, liver, lung and bones. However this case is particular because widespread disease was diagnosed without lymph node involvement, suggesting an hematogenous diffusion. The median age of patients with eye metastases from bladder cancer reported so far is 58 years (range, 43-75) (Table I). In the majority of cases, eye involvement is metachronous and is associated to other metastatic sites (Table I). Five of 24 cases are reported as synchronous metastases but 4 out of 19 described as metachronous show a time to eye metastases less than 1 month. At the light of this observation, it should be recommended a careful and correct staging of the disease at the time of diagnosis. In fact, in patients with ocular symptoms the probability of eye involvement should be considered, even if it is uncommon. Clinical presentation may differ depending on ocular or orbital localization and it could be peculiar for each metastatic site (5). In fact exophthalmos, proptosis and pain are likely related to orbital involvement. Moreover, as reported in literature, other signs or symptoms like visual disorders, diplopia, paralysis of the VI cranial nerve, hyperesthesia of the trigeminal nerve, amaurosis, inflamed conjunctiva, retinal detachment and even palpable mass should not be underestimated. Because of the rarity of eye involvement, there is no diagnostic algorithm: Specific clinical visit and CT scan are recommended and differential diagnosis includes: Retro bulbar region or lacrimal gland tumors, retinal abscess, metastasis from carcinoma, and granuloma (25). In this patient, retro-bulbar region biopsy was performed because of visual disorders that occurred before liver involvement diagnosis. Only few patients among the cases reported in 


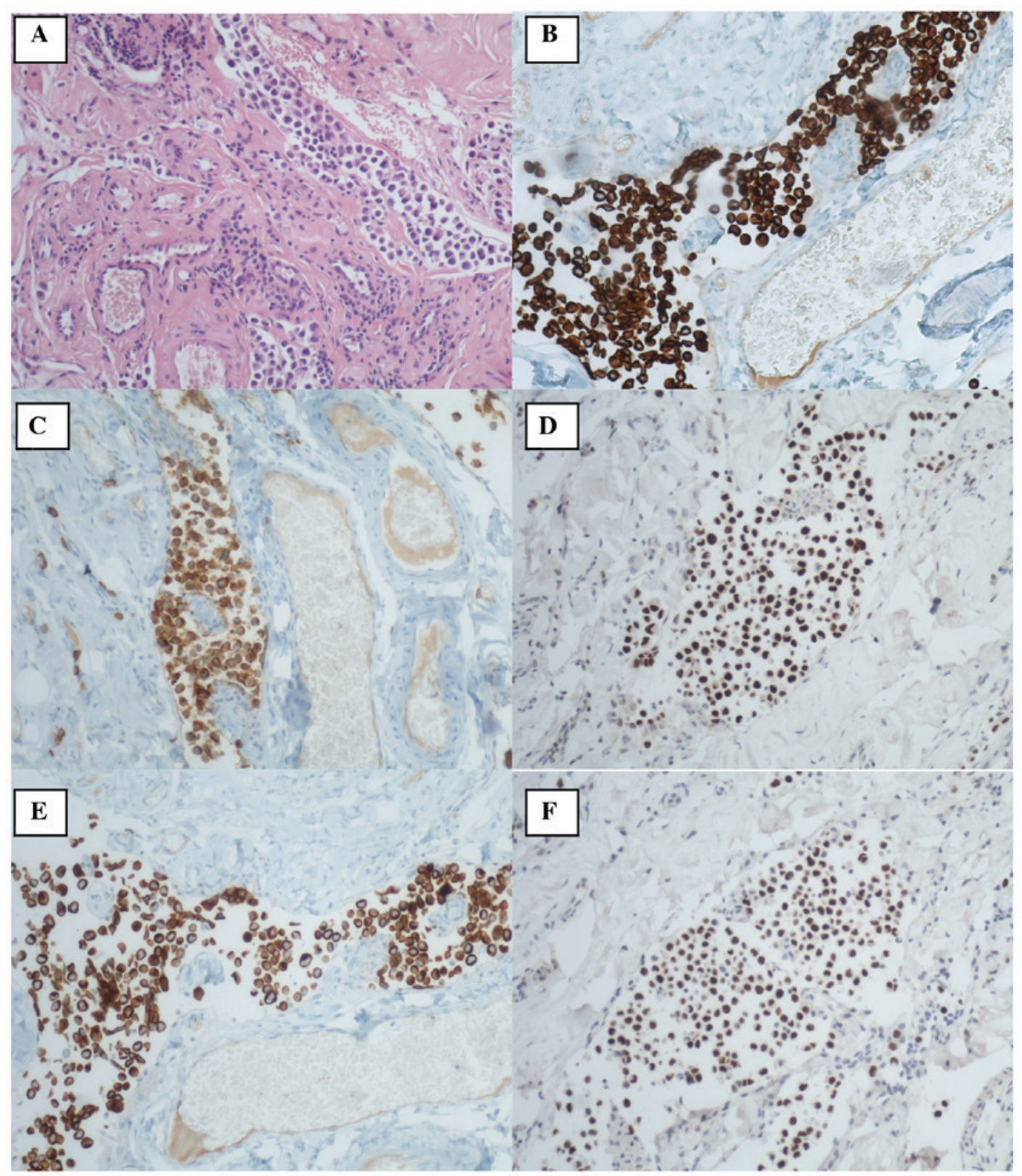

Figure 2. Hematoxylin and eosin examination revealed (A) neoplastic infiltration of fibro-adipose tissue characterized by diffuse population of cellular elements with a high eosinophilic cytoplasm and eccentric nuclei. Immunohistochemistry showed positive staining for (B) CK7, (C) Cluster of Differentiation-138, (D) GATA3, (E) CK20 and (F) dnp63. Magnification, x400. CK, cytokeratin.

literature had received a cytological or histological examination. Immunohistochemical staining (IHC) of the nuclei of the tumor cells was intensely positive for transcription factor GATA3, which is specific for urothelial and breast carcinoma. The cells were also strongly immunoreactive for epithelial markers CKAE1/AE3, CK5, CK7 and CK20. Co-expression of CK7 and CK20 is a differential feature of urothelial carcinoma and excludes breast carcinoma that is positive only for CK7. Positive CD138, DNP63 and negative LCA, CD79 $\alpha$ completed the diagnosis. Metastatic eye involvement indicates a later stage of the disease with an extremely poor prognosis. In the literature, median time to eye metastases is 11 months (Table I) and the median overall survival is 3 months (Fig. 3). In the present case, no chemotherapy was administered due to the poor ECOG PS and liver impairment showed by laboratory examinations.
There is no specific treatment for eye metastases and the aim of the cure is palliative. Orbital and ocular metastatic sites could receive theoretically different approaches but treatment should be evaluated case by case because of the lack of evidences. Only 5 out of 24 patients reported so far received local radiotherapy and 1 out of 24 received surgical decompression in order to improve the ocular symptoms. Conversely, the majority of patients received chemotherapy for metastatic disease without clinical or survival benefit.

To the best of our knowledge, this is the first qualitative systematic review about eye metastases caused by urothelial bladder cancer. Furthermore, the case described in this article represents the first report of retro-bulbar involvement that infiltrates the lacrimal gland from urothelial bladder cancer. Another novelty of this case is represented by an accurate IHC evaluation in order to clarify the differential diagnoses 
Table I. Summary of reported cases of urothelial-bladder cancer metastasizing to the eye.

\begin{tabular}{|c|c|c|c|c|c|c|c|c|}
\hline Author, year & $\begin{array}{c}\text { Age } \\
\text { (years) }\end{array}$ & Gender & $\begin{array}{c}\text { Eye } \\
\text { localization }\end{array}$ & $\begin{array}{l}\text { Time to } \\
\text { ocular } \\
\text { metastasis } \\
\text { (months) }\end{array}$ & Treatment & $\begin{array}{l}\text { Treatment } \\
\text { for ocular } \\
\text { metastasis }\end{array}$ & $\begin{array}{c}\text { OS } \\
\text { (months) }\end{array}$ & (Refs.) \\
\hline Smiley, 1965 & 75 & $\mathrm{M}$ & Retrobulbar, L & Synchronous & BSC & No & 8 & (7) \\
\hline Resnick et al, 1975 & 46 & $\mathrm{M}$ & Orbit, L & 1 & ADRIA+5FU & No & 4 & (8) \\
\hline Resnick et al, 1975 & 51 & $\mathrm{M}$ & Choroid, R & 15 & No & RT & 4 & (8) \\
\hline Cieplinski et al, 1982 & 57 & M & Choroid, B & 8 & $\begin{array}{l}\text { CDDP+ } \\
\text { ADRIA+CTX }\end{array}$ & RT & 0.5 & (9) \\
\hline Krauss et al, 1982 & 64 & $\mathrm{~F}$ & Orbit, R & 15 & No & RT & 1 & (10) \\
\hline Prats et al, 1989 & 58 & M & Orbit, L & Synchronous & No & $\begin{array}{l}\text { Surgical } \\
\text { decompression }\end{array}$ & 4 & (11) \\
\hline Felip et al, 1991 & 58 & M & Orbit & 0,5 & NA & No & 3 & (12) \\
\hline Felip et al, 1991 & 62 & M & Orbit & 36 & NA & No & LF & (12) \\
\hline Angulo et al, 1991 & 61 & M & Retro-orbital & 11 & $\mathrm{BSC}$ & No & 0.9 & (13) \\
\hline Hugkulstone et al, 1994 & 45 & M & Orbit, B & Synchronous & MVAC & RT & 5 & (14) \\
\hline Scott and Williams, 1994 & NA & NA & Orbit & Synchronous & NA & NA & 8 & (15) \\
\hline $\begin{array}{l}\text { Fynn-Thompson et al, } \\
2003\end{array}$ & 68 & M & $\begin{array}{l}\text { Orbital + } \\
\text { optic nerve, L }\end{array}$ & 48 & $\mathrm{BSC}$ & No & 1 & (16) \\
\hline Nabi et al, 2002 & 43 & $\mathrm{~F}$ & Optic canal L & 6 & MVAC & No & 3 & (17) \\
\hline Nabi et al, 2002 & 79 & M & Choroid R & 8 & MVAC & No & 1.6 & $(17)$ \\
\hline Amemiya et al, 2002 & 55 & M & Orbital B & NA & NA & NA & NA & (18) \\
\hline Amemiya et al, 2002 & 73 & M & Orbital R & NA & NA & NA & NA & (18) \\
\hline Souza Filho et al, 2005 & 53 & M & Orbit L & 3 weeks & $\mathrm{BSC}$ & no & 1 & (19) \\
\hline Levecq et al, 2007 & 58 & M & Choroidal & 96 & no & $\begin{array}{l}\text { Enucleation, } \\
\text { RT }\end{array}$ & 7 & (20) \\
\hline Lin et al, 2007 & 60 & M & Orbit R & 8 & MVAC & No & $\begin{array}{l}\text { Alive } \\
\text { at } 6\end{array}$ & (4) \\
\hline Wettach and Steele 2008 & 66 & M & Orbit R & Synchronous & CBDCA+GEM & No & 1 & (21) \\
\hline Mitsui et al, 2014 & 48 & M & Choroid R & 17 & MVAC & No & 5 & $(2)$ \\
\hline SooHoo et al, 2012 & 53 & $\mathrm{~F}$ & Orbit R & 1 & $\mathrm{BSC}$ & No & 3 & $(22)$ \\
\hline Magrath et al, 2015 & 57 & $\mathrm{~F}$ & Orbit L & Synchronous & $\mathrm{BSC}$ & No & $\mathrm{LF}$ & (23) \\
\hline Present case & 70 & M & $\begin{array}{l}\text { Retrobulbar R, } \\
\text { lacrimal gland }\end{array}$ & 33 & $\mathrm{BSC}$ & No & 3 & - \\
\hline
\end{tabular}

NA, not available; M, male; F, female; L, left; R, right; B, bilateral; BSC, best supportive care; ADRIA, adriamycin; 5FU, 5-fluoroiracil; CDDP, cisplatin; CTX, cyclophosphamide; MVAC, methotrexate + vinblastine + adriamycin + cisplatin; CBDCA, carboplatin; GEM, gemcitabine; RT, radiotherapy; OS, overall survival; LF, lost to follow up.

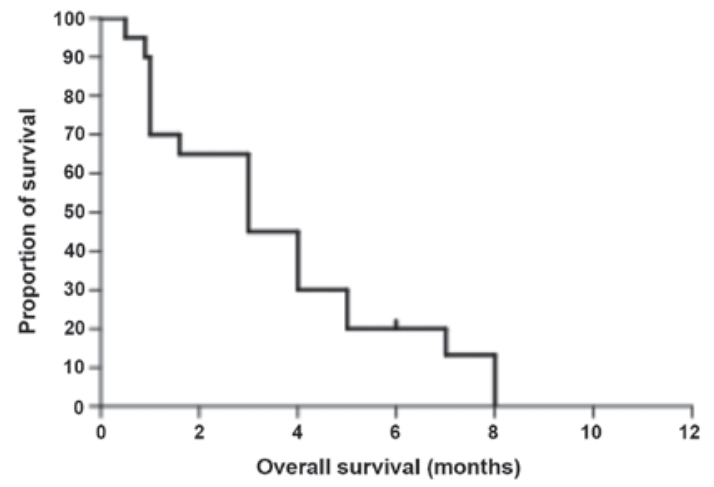

Figure 3. Kaplan Meier curve for overall survival from the occurrence of ocular metastasis. among the different primary tumor sites. Muscular infiltrative bladder cancer should be considered as a potential micro metastatic disease since the beginning. Therefore, Oncologist should be aware about the possibility of eye involvement in patients with a past medical history of urothelial bladder cancer associated with eye disorders. Multidisciplinary approach is required for an early detection of this condition that should lead to a prompt therapeutic intervention. The rarity of metastatic eye localization does not provide high level of evidence for a specific treatment. Actually, majority of patients receive chemotherapy with poor outcomes. Molecular and biological aspects of both urothelial bladder cancer and metastatic sites should be further investigated in order to improve therapeutic options and patients' prognosis. 


\section{Acknowledgements}

Not applicable.

\section{Funding}

The present study was funded by SAMAR Laboratorio di Istopatologia di Roma.

\section{Availability of data and materials}

The datasets used and/or analyzed during the current study are available from the corresponding author on reasonable request.

\section{Authors' contributions}

GG, NO and PP conceived the research, wrote the paper, and assessed the figures and tables. LC and EDS collected the case report data. LC, EM, AR and $\mathrm{MB}$ reviewed and confirmed the histological diagnosis. MP, GC, TDR, MRDA and AS performed the literature research and critically reviewed the manuscript for important intellectual content. PP and GG supervised the project.

\section{Ethics approval and consent to participate}

The Ethics Committee 'LAZIO 1' n. 2001/2017 (Rome, Italy; prot. no. 2198) approved all procedures.

\section{Patient consent for publication}

The patient provided written informed consent for publication of any associated data and accompanying images.

\section{Competing interests}

The authors declare that they have no competing interests.

\section{References}

1. Burger M, Catto JW, Dalbagni G, Grossman HB, Herr H, Karakiewicz P, Kassouf W, Kiemeney LA, La Vecchia C, Shariat S and Lotan Y: Epidemiology and risk factors of urothelial bladder cancer. Eur Urol 63: 234-241, 2013.

2. Mitsui Y, Arichi N, Inoue K, Hiraki M, Nakamura S, Hiraoka T, Ishikawa N, Maruyama R, Yasumoto H and Shiina H: Choroidal and cutaneous metastasis from urothelial carcinoma of the bladder after radical cystectomy: A case report and literature review. Case Rep Urol 2014: 491541, 2014.

3. Cohen VM: Ocular metastases. Eye (Lond) 27: 137-141, 2013.

4. Lin HC, Chang CH, Li WM, Hsiao HL, Chang TH, Wu WJ and Huang CH: Orbital metastasis from urothelial carcinoma of the urinary bladder. Kaohsiung J Med Sci 23: 84-88, 2007.
5. Bita E (ed): Ophthalmic Oncology. In: MD Anderson Solid Tumor Oncology Series. Spinger, New York, NY, 2011.

6. Goldberg RA, Rootman J and Cline R: Tumors metastatic to the orbit: A changing picture. Surv Ophthalmol 35: 1-24, 1990.

7. Smiley SS: An orbital metastasis from the urinary bladder. Arch Opthalmol 74: 809-810, 1965

8. Resnick MI, O'Conor VJ Jr and Grayhack JT: Metastases to the eye from transitional cell carcinoma of the bladder. J Urol 114: 722-724, 1975

9. Cieplinski W, Ciesielski TE, Haine C and Nieh P: Choroid metastases from transitional cell carcinoma of the bladder. A case report and a review of the literature. Cancer 50: 1596-1600, 1982.

10. Krauss HR, Slamovits TL, Sibony PA, Dekker A and Kennerdell JS: Orbital metastasis of bladder carcinoma. Am J Ophthalmol 94: 265-267, 1982.

11. Prats J, Bellmunt J, Calvo MA, Sarrias F and Toran N: Orbital metastasis, by transitional cell carcinoma of the bladder. Int Urol Nephrol 21: 389-392, 1989.

12. Felip E, Rovirosa MA, Salud A, Capdevila F, Bellmunt J and Giralt J: Orbital metastases from transitional-cell cancer of the urinary bladder. Urol Int 46: 82-84, 1991.

13. Angulo JC, Lopez JI, Larrinaga JR and Flores N: Metastasising carcinoma of the urinary bladder presenting as a retro-orbital mass. Case report. Scand J Urol Nephrol 25: 83-84, 1991.

14. Hugkulstone CE, Winder S and Sokal M: Bilateral orbital metastasis from transitional cell carcinoma of the bladder. Eye (Lond) 8: 580-582, 1994.

15. Scott JA and Williams R: Orbital metastasis from bladder carcinoma. Eye 6: 664-666, 1994.

16. Fynn-Thompson N, McKiernan JM and Fay A: Transitional cell carcinoma of the urinary bladder metastatic to the orbit. Ophthalmic Plast Reconstr Surg 19: 165-167, 2003.

17. Nabi G, Dadeya S, Dogra PN and Lal H: Eye metastasis form urothelial tumours. Int Urol Nephrol 34: 51-54, 2002.

18. Amemiya T, Hayashida $\mathrm{H}$ and Dake Y: Metastatic orbital tumors in Japan: A review of the literature. Ophthalmic Epidemiol 9: 35-47, 2002.

19. Souza Filho JP, Odashiro AN, Pereira PR, Al-Buloushi A, Codere $\mathrm{F}$ and Burnier MN: Orbital metastasis of urinary bladder carcinoma: A clinicopathologic report and review of the literature. Orbit 24: 269-271, 2005.

20. Levecq L, De Potter P, Godfraind C, Guagnini AP and Kozyreff A: Choroidal metastasis from carcinoma of the bladder. Retin Cases Brief Rep 1: 251-253, 2007.

21. Wettach GR and Steele EA: Urothelial cell carcinoma of the bladder presenting as orbital metastasis. Arch Pathol Lab Med 132: 1224, 2008.

22. SooHoo JR, Gonzalez MO, Siomos VJ and Durairaj VD: Urothelial carcinoma with orbital metastasis. Urology 80: e45-e46, 2012.

23. Magrath GN, Proctor CM, Reardon WA, Patel KG, Lentsch EJ and Eiseman AS: Esophageal adenocarcinoma and urothelial carcinoma orbital metastases masquerading as infection. Orbit 34: 51-55, 2015.

24. Moher D, Liberati A, Tetzlaff J and Altman DG; The PRISMA group: Preferred reporting items for systematic reviews and meta-analyses: PRISMA statement. Ann Intern Med 151: 264-269, 2009.

25. Shields JA, Bakewell B, Augsburger JJ and Flanagan JC: Classification and incidence of space-occupying lesions of the orbit. A survey of 645 biopsies. Arch Ophthalmol 102: 1606-1611, 1984.

26. Konstantinidis L and Damato B: Intraocular metastases-a review. Asia Pac J Ophthalmol (Phila) 6: 208-214, 2017. 